\title{
RMetS
}

Royal Meteorological Society

\section{Hydrostatic Hamiltonian particle-mesh (HPM) methods for atmospheric modelling}

\author{
Seoleun Shin, ${ }^{\text {a }}$ Sebastian Reich ${ }^{\mathrm{a}}$ and Jason Frank ${ }^{\mathrm{b}}$ \\ anstitut für Mathematik, Universität Potsdam, Potsdam, Germany \\ ${ }^{\mathrm{b}} \mathrm{CWI}$, Amsterdam, the Netherlands \\ ${ }^{\star}$ Correspondence to: S. Shin, Institut für Mathematik, University of Potsdam, Am Neuen Palais 10, D-14469 Potsdam, \\ Germany. E-mail: seshin@uni-potsdam.de
}

\begin{abstract}
We develop a hydrostatic Hamiltonian particle-mesh (HPM) method for efficient long-term numerical integration of the atmosphere. In the HPM method, the hydrostatic approximation is interpreted as a holonomic constraint for the vertical position of particles. This can be viewed as defining a set of vertically buoyant horizontal meshes, with the altitude of each mesh point determined so as to satisfy the hydrostatic balance condition and with particles modelling horizontal advection between the moving meshes. We implement the method in a vertical-slice model and evaluate its performance for the simulation of idealized linear and nonlinear orographic flow in both dry and moist environments. The HPM method is able to capture the basic features of the gravity wave to a degree of accuracy comparable with that reported in the literature. The numerical solution in the moist experiment indicates that the influence of moisture on wave characteristics is represented reasonably well and the reduction of momentum flux is in good agreement with theoretical analysis. Copyright (C) 2011 Royal Meteorological Society
\end{abstract}

Key Words: conservative discretization; Lagrangian modeling; holonomic constraints; fluid mechanics

Received 17 March 2011; Revised 19 August 2011; Accepted 7 November 2011; Published online in Wiley Online Library

Citation: Shin S, Reich S, Frank J. 2011. Hydrostatic Hamiltonian particle-mesh (HPM) methods for atmospheric modelling. Q. J. R. Meteorol. Soc. DOI:10.1002/qj.982

\section{Introduction}

In recent years efforts have been made to extend the use of Hamiltonian particle-mesh (HPM) methods for atmospheric modelling (Frank et al., 2002; Frank and Reich, 2004; Cotter et al., 2004; Shin and Reich, 2009). An important issue in atmospheric modelling, particularly for climate simulation, is the numerical conservation of mass of air, water and long-lived tracers (Thuburn, 2008). The hydrostatic HPM method has some advantages with respect to this issue in that it conserves mass locally and satisfies an exact advection equation for long-term simulations. The key idea is to approximate horizontal motion along the lines of the Hamiltonian particle-mesh method, while the vertical motion is discretized as a moving-mesh method. Lin (2004) has also discussed the reduction of dimensionality from $3 \mathrm{D}$ to layered $2 \mathrm{D}$ by using floating vertical coordinates in the context of a finite-volume dynamical core. In this article we present the hydrostatic HPM method and test the scheme for idealized orographic flows. We demonstrate that the HPM is able to represent fundamental processes, such as the generation of gravity waves due to orographic forcing and the influence of moist processes on the character of idealized linear and nonlinear orographic waves, properly. Compared with previous numerical studies by Durran and Klemp (1983, hereafter DK83) and Pinty et al. (1995, hereafter P95) for linear hydrostatic orographic flows with an isolated hill of $1 \mathrm{~m}$ height, the wave generation is well represented in our model.

In the following sections we explain the details of the HPM method and how we represent moving meshes to enforce time-dependent hydrostatic balance. Then we discuss the results of numerical experiments and propose further work with the HPM method. 


\section{The hydrostatic HPM}

The hydrostatic HPM method is derived for a vertical slice model, using Eulerian coordinates $(x, z) \in \Omega \subset \mathbb{R}^{2}$ and Lagrangian labels $(a, c) \in \Omega$. We denote discrete approximations of dependent variables over the Eulerian grid with Latin indices, and those over a discrete label space with Greek indices. For example, $f_{\alpha, \gamma}$ is an approximation to $f(a, c)$ at a grid point $\left(a_{\alpha}, c_{\gamma}\right)=(\alpha \Delta a, \gamma \Delta c)$ in label space, where $\Delta a, \Delta c$ are mesh sizes and $\alpha, \gamma \in \mathbb{Z}$. For mixed Eulerian-Lagrangian approximations, we use a form such as $f_{i, \gamma} \approx f\left(x_{i}, c_{\gamma}\right)$. More details about such mixed approximations will be given in the following paragraphs.

The hydrostatic HPM method is different in nature from a non-hydrostatic HPM (Shin and Reich, 2009) in that the vertical motion is discretized using a moving mesh. We define time-dependent Lagrangian particles with $x$ positions denoted by $x_{\alpha, \gamma+1 / 2}(t)$, and vertically buoyant mesh points with $z$-coordinates $z_{i, \gamma}(t)$. As indicated by the indexing, the horizontal position of the mesh point is fixed in the Eulerian frame, while its vertical position is fixed in the Lagrangian frame, i.e. $z=z(x, c)$. We define horizontal basis functions with support centred at the mean of two vertically moving mesh points, i.e. at $\left(x_{i}, z_{i, \gamma+1 / 2}\right)$ with $z_{i, \gamma+1 / 2}=\left(z_{i, \gamma+1}+z_{i, \gamma}\right) / 2$. The basis functions, denoted by $\psi_{i, \gamma+1 / 2}(x)$, are non-negative and satisfy the partition of unity property:

$$
\sum_{i} \psi_{i, \gamma+1 / 2}(x)=1
$$

for all $(x, z) \in \Omega$. We also introduce the scaled basis functions:

$$
\hat{\psi}_{i, \gamma+1 / 2}(x)=\frac{\psi_{i, \gamma+1 / 2}(x)}{\Delta x} .
$$

It is natural to use the same basis function at $x_{i}$, independent of the value of $\gamma$. Hence we will use the simpler notation $\psi_{i}(x)$ and $\hat{\psi}_{i}(x)$. A basis function having the desired properties is the cubic B-spline with support radius $2 \Delta x$.

A Lagrangian particle with $x$ position $x_{\alpha, \gamma+1 / 2}$ carries a mass $m_{\alpha, \gamma+1 / 2}$ and a potential temperature $\theta_{\alpha, \gamma+1 / 2}$. Note that we do not request that $\theta_{\alpha, \gamma+1 / 2}=\theta_{\gamma+1 / 2}$, i.e. lines of constant $c_{\gamma+1 / 2}$ need not be lines of constant potential temperature (isentropes). Additionally, in the case of moist simulations, a particle also carries a moisture budget, which is commonly represented by the total mixing ratio $r_{t \alpha, \gamma+1 / 2}$. The moisture can experience phase changes and in this study we consider only those between cloud water and water vapour. The three quantities $\left(m_{\alpha, \gamma+1 / 2}, \theta_{\alpha, \gamma+1 / 2}, r_{t_{\alpha, \gamma+1 / 2}}\right)$ remain constant under unsaturated conditions, but $\theta_{\alpha, \gamma+1 / 2}$ may vary when latent heat is released in association with phase changes of water. The quantities $m_{\alpha, \gamma+1 / 2}$ and $r_{t_{\alpha, \gamma+1 / 2}}$ always remain constant. Regardless of the saturated/unsaturated condition, moist static energy is conserved so that the scheme can be energetically consistent.

Here we consider simple problems of air flows in hydrostatic balance, which are a good approximation for orographic flow arising due to moderate surface terrain.

\subsection{Density approximation}

The density $\rho_{i, \gamma+1 / 2}(t) \approx \rho\left(x_{i}, z_{i, \gamma+1 / 2}\right)$ is approximated by

$$
\begin{aligned}
& \rho_{i, \gamma+1 / 2}(t) \\
& =\frac{1}{z_{i, \gamma+1}(t)-z_{i, \gamma}(t)}\left[\sum_{\alpha} m_{\alpha, \gamma+1 / 2} \hat{\psi}_{i}\left(x_{\alpha, \gamma+1 / 2}(t)\right)\right] .
\end{aligned}
$$

Conservation of mass is encoded in the identity

$$
\begin{aligned}
& \sum_{i, \gamma} \rho_{i, \gamma+1 / 2}(t) \Delta x\left[z_{i, \gamma+1}(t)-z_{i, \gamma}(t)\right] \\
= & \sum_{i, \gamma}\left[\sum_{\alpha} m_{\alpha, \gamma+1 / 2} \hat{\psi}_{i}\left(x_{\alpha, \gamma+1 / 2}(t)\right)\right] \Delta x \\
= & \sum_{\alpha, \gamma} m_{\alpha, \gamma+1 / 2}\left[\sum_{i} \psi_{i}\left(x_{\alpha, \gamma+1 / 2}(t)\right)\right] \\
= & \sum_{\alpha, \gamma} m_{\alpha, \gamma+1 / 2}
\end{aligned}
$$

due to (2.1). The product of density and potential temperature, i.e. $\mu=\theta \rho$, is a primitive variable approximated at (vertically moving) mesh points $\left(x_{i}, z_{i, \gamma+1 / 2}\right)$. The approximation for $\mu_{i, \gamma+1 / 2}$ is

$$
\begin{aligned}
\mu_{i, \gamma+1 / 2}(t) & =\frac{1}{z_{i, \gamma+1}(t)-z_{i, \gamma}(t)} \\
& {\left[\sum_{\alpha} \theta_{\alpha, \gamma+1 / 2} m_{\alpha, \gamma+1 / 2} \hat{\psi}_{i}\left(x_{\alpha, \gamma+1 / 2}(t)\right)\right], }
\end{aligned}
$$

and we obtain conservation via

$$
\begin{aligned}
\sum_{i, \gamma} \mu_{i, \gamma+1 / 2}(t) & \Delta x\left[z_{i, \gamma+1}(t)-z_{i, \gamma}(t)\right] \\
= & \sum_{\alpha, \gamma} \theta_{\alpha, \gamma+1 / 2} m_{\alpha, \gamma+1 / 2} .
\end{aligned}
$$

Other thermodynamic quantities are now easily approximated over $\left(x_{i}, z_{i, \gamma+1 / 2}\right)$ using the standard identities

$$
T=\theta \pi, \quad \pi=\left(\frac{\mu}{\mu_{0}}\right)^{\kappa /(1-\kappa)},
$$

where $\mu_{0}=p_{0} / R$ is a constant reference value, $c_{\mathrm{p}}=$ $c_{\mathrm{v}} /(1-\kappa)$ and $\kappa=R_{\mathrm{d}} / c_{\mathrm{p}}=2 / 7$, where $R_{\mathrm{d}}$ is the ideal gas constant for dry air.

\subsection{Equations of motion and discrete hydrostatic balance}

Since we work with the hydrostatic approximation, the vertical motion does not contribute to the kinetic energy, the discrete approximation for which is

$$
\mathcal{T}=\frac{1}{2} \sum_{\alpha, \gamma} m_{\alpha, \gamma+1 / 2}\left|\dot{x}_{\alpha, \gamma+1 / 2}\right|^{2} .
$$


The potential energy is defined by

$$
\mathcal{V}_{\text {analytic }}=\int_{\Omega}\left[c_{\mathrm{v}} \mu_{0}\left(\frac{\mu}{\mu_{0}}\right)^{1 /(1-\kappa)}+g \rho z\right] \mathrm{d} x \mathrm{~d} z .
$$

With our given approximations, this becomes

$$
\begin{aligned}
\mathcal{V}=\sum_{i, \gamma} & {\left[c_{\mathrm{v}} \mu_{0}\left(\frac{\mu_{i, \gamma+1 / 2}}{\mu_{0}}\right)^{1 /(1-\kappa)}\right.} \\
& \left.+g \rho_{i, \gamma+1 / 2} z_{i, \gamma+1 / 2}\right] \Delta x \Delta z_{i, \gamma+1 / 2}=\mathcal{V}^{\mathrm{P}}+\mathcal{V}^{\mathrm{G}}
\end{aligned}
$$

where for ease of notation we introduce the following abbreviation:

$$
\Delta z_{i, \gamma+1 / 2}=z_{i, \gamma+1}-z_{i, \gamma}
$$

The continuous equations of motion are now obtained from the Lagrangian functional

$$
L=\int \mathcal{L} \mathrm{d} t
$$

where $\mathcal{L}=\mathcal{T}-\mathcal{V}$. The associated discrete Euler-Lagrange equations are straightforward to derive. At time level $t_{n}$, the contribution to the Lagrangian is

$$
\begin{gathered}
\mathcal{L}^{n}\left(x_{\alpha, \gamma+1 / 2}^{n}\right)=\frac{1}{2} m_{\alpha, \gamma+1 / 2}\left(\frac{x_{\alpha, \gamma+1 / 2}^{n}-x_{\alpha, \gamma+1 / 2}^{n-1}}{\Delta t}\right)^{2} \\
-\mathcal{V}\left(x_{\alpha, \gamma+1 / 2}^{n}\right) .
\end{gathered}
$$

The fully discrete approximation of the action integral $L$ is then given by

$$
L_{\Delta t}=\sum_{n=1}^{N} \mathcal{L}^{n} \Delta t
$$

Computing partial derivatives of $L_{\Delta t}$ with respect to $x_{\alpha, \gamma+1 / 2}^{n}$, and setting them equal to zero, we obtain

$$
\begin{aligned}
0= & \frac{\partial L_{\Delta t}}{\partial x_{\alpha, \gamma+1 / 2}^{n}} \\
=-m_{\alpha, \gamma+1 / 2} \frac{\left(x_{\alpha, \gamma+1 / 2}^{n+1}-2 x_{\alpha, \gamma+1 / 2}^{n}+x_{\alpha, \gamma+1 / 2}^{n-1}\right)}{\Delta t} & -\Delta t \nabla_{x} \mathcal{V}\left(x_{\alpha, \gamma+1 / 2}^{n}\right) .
\end{aligned}
$$

This equation is the discrete analogue of the Euler-Lagrangian equation

$$
\frac{\mathrm{d}}{\mathrm{d} t} \frac{\partial \mathcal{L}}{\partial \dot{x}}=\frac{\partial \mathcal{L}}{\partial x} .
$$

The momentum equation is given by

$$
\begin{aligned}
m_{\alpha, \gamma+1 / 2} \ddot{x}_{\alpha, \gamma+1 / 2}=- & \nabla_{x} \mathcal{V}\left(x_{\alpha, \gamma+1 / 2}^{n}\right) \\
& =F_{x, \alpha, \gamma+1 / 2}^{\mathrm{G}}+F_{x, \alpha, \gamma+1 / 2}^{\mathrm{P}},
\end{aligned}
$$

where the gravitational force applied on the particle is

$$
F_{x, \alpha, \gamma+1 / 2}^{\mathrm{G}}=-g m_{\alpha, \gamma+1 / 2} \sum_{i}\left[z_{i, \gamma+1 / 2} \nabla_{x} \psi_{i}\left(x_{\alpha, \gamma+1 / 2}\right)\right],
$$

and the pressure gradient force is

$$
\begin{aligned}
& F_{x, \alpha, \gamma+1 / 2}^{\mathrm{P}}=-c_{\mathrm{p}} m_{\alpha, \gamma+1 / 2} \theta_{\alpha, \gamma+1 / 2} \\
& \quad \times \sum_{i}\left[\left(\frac{\mu_{i, \gamma+1 / 2}}{\mu_{0}}\right)^{\kappa /(1-\kappa)} \nabla_{x} \psi_{i}\left(x_{\alpha, \gamma+1 / 2}\right)\right] .
\end{aligned}
$$

Störmer-Verlet time-stepping equivalent to (2.15) leads to the explicit update

$$
\begin{array}{r}
m_{\alpha, \gamma+1 / 2}\left[x_{\alpha, \gamma+1 / 2}^{n+1}-2 x_{\alpha, \gamma+1 / 2}^{n}+x_{\alpha, \gamma+1 / 2}^{n+1}\right] \\
=\Delta t^{2}\left[F_{x, \alpha, \gamma+1 / 2}^{\mathrm{G}, n}+F_{x, \alpha, \gamma+1 / 2}^{\mathrm{P}, n}\right] .
\end{array}
$$

Since $\mathcal{L}$ does not depend on $\dot{z}_{i, \gamma+1 / 2}$, the associated Euler-Lagrange equation for $z$ results in a holonomic constraint, which on the discrete level becomes

$$
0=\frac{\partial L_{\Delta t}}{\partial z_{i, \gamma+1 / 2}^{n}}=-\Delta t \nabla_{z} \mathcal{V}\left(z_{i, \gamma+1 / 2}^{n}\right) .
$$

These nonlinear constraint equations are coupled in the index $\gamma$ but not in $i$. Hence we essentially have to solve a decoupled set of discretized, one-dimensional, nonlinear elliptic equations in the vertical. Note that this also holds true in three dimensions. The appropriate boundary conditions are $z_{i, 0}=0$ and $z_{i, M}=H$ for the bottom and top surfaces. It is easy to include orography into this approach by simply setting $z_{i, 0}=z_{\mathrm{s}}\left(x_{i}\right)$, where $z_{\mathrm{s}}\left(x_{i}\right)$ is the height of the surface at $x_{i}$.

To simplify notation in the following, we define $\bar{\mu}_{i, \gamma+1 / 2}=\mu_{i, \gamma+1 / 2} \Delta z_{i, \gamma+1 / 2}$ and $\bar{\rho}_{i, \gamma+1 / 2}=\rho_{i, \gamma+1 / 2} \Delta z_{i, \gamma+1 / 2}$. To complete the time-step at $t_{n+1}$, we obtain the new vertical positions $z_{i, \gamma}^{n+1}$ that satisfy the discrete hydrostatic balance condition. Equation (2.21) becomes

$$
0=F_{z, i, \gamma}^{\mathrm{G}}+F_{z, i, \gamma}^{\mathrm{P}}
$$

with

$$
F_{z, i, \gamma}^{\mathrm{G}}=-\frac{g}{2}\left[\bar{\rho}_{i, \gamma+1 / 2}+\bar{\rho}_{i, \gamma-1 / 2}\right]
$$

and

$$
\begin{aligned}
F_{z, i, \gamma}^{\mathrm{P}}= & c_{\mathrm{v}} \mu_{0}\left[\left(\frac{\mu_{i, \gamma+1 / 2}}{\mu_{0}}\right)^{1 /(1-\kappa)}-\left(\frac{\mu_{i, \gamma-1 / 2}}{\mu_{0}}\right)^{1 /(1-\kappa)}\right] \\
& -c_{\mathrm{p}}\left[\mu_{i, \gamma+1 / 2}\left(\frac{\mu_{i, \gamma+1 / 2}}{\mu_{0}}\right)^{\kappa /(1-\kappa)}\right] \\
& \left.-\mu_{i, \gamma-1 / 2}\left(\frac{\mu_{i, \gamma-1 / 2}}{\mu_{0}}\right)^{\kappa /(1-\kappa)}\right] \\
= & -\kappa c_{\mathrm{p}} \mu_{0}\left[\left(\frac{\mu_{i, \gamma+1 / 2}}{\mu_{0}}\right)^{1 /(1-\kappa)}-\left(\frac{\mu_{i, \gamma-1 / 2}}{\mu_{0}}\right)^{1 /(1-\kappa)}\right],
\end{aligned}
$$

by again applying the discrete variational principle. These equations are solved subject to the boundary conditions $z_{i, 0}$ $=z_{\mathrm{s}}\left(x_{i}\right)$ and $z_{i, M}=H$. Note that with particles horizontally fixed, $F_{z, i, \gamma}^{\mathrm{G}}$ is constant. We use the relation

$$
\begin{aligned}
F_{z, i, \gamma}^{\mathrm{P}}= & -\kappa c_{\mathrm{p}} \mu_{0}\left[\left(\frac{\bar{\mu}_{i, \gamma+1 / 2}}{\mu_{0}}\right)^{1 /(1-\kappa)} \Delta z_{i, \gamma+1 / 2}^{1 /(\kappa-1)}\right. \\
& \left.-\left(\frac{\bar{\mu}_{i, \gamma-1 / 2}}{\mu_{0}}\right)^{1 /(1-\kappa)} \Delta z_{i, \gamma-1 / 2}^{1 /(\kappa-1)}\right]
\end{aligned}
$$


and

$$
\begin{aligned}
\frac{\partial}{\partial z_{i, \gamma}} \Delta z_{i, \gamma+1 / 2}^{1 /(\kappa-1)}=\frac{\partial}{\partial z_{i, \gamma}}\left(z_{i, \gamma+1}-z_{i, \gamma}\right)^{1 /(\kappa-1)} \\
=\frac{1}{1-\kappa} \Delta z_{i, \gamma+1 / 2}^{1 /(\kappa-1)} \Delta z_{i, \gamma+1 / 2}^{-1}
\end{aligned}
$$

plus related formulae to set up a Newton iteration for finding $z_{i, \gamma+1 / 2}$. The vertical position of the particle at $x_{\alpha, \gamma+1 / 2}$ is then approximated by

$$
z_{\alpha, \gamma+1 / 2}=\sum_{i} z_{i, \gamma+1 / 2} \psi_{i}\left(x_{\alpha, \gamma+1 / 2}(t)\right)
$$

and the diagnostic estimation of the vertical velocity can be given by

$$
W_{\alpha, \gamma+1 / 2}^{n+1}=\frac{1}{\Delta t}\left(z_{\alpha, \gamma+1 / 2}^{n+1}-z_{\alpha, \gamma+1 / 2}^{n}\right),
$$

while we denote the zonal velocity of a particle, $\dot{x}_{\alpha, \gamma+1 / 2}$ by $U_{\alpha, \gamma+1 / 2}$. The grid-based zonal and vertical winds are then defined by

$$
\begin{aligned}
u_{i, \gamma+1 / 2} & =\frac{\sum_{\alpha} U_{\alpha, \gamma+1 / 2} m_{\alpha, \gamma+1 / 2} \psi_{i}\left(x_{\alpha, \gamma+1 / 2}\right)}{\rho_{i, \gamma+1 / 2} \Delta x \Delta z_{i, \gamma+1 / 2}}, \\
w_{i, \gamma+1 / 2} & =\frac{\sum_{\alpha} W_{\alpha, \gamma+1 / 2} m_{\alpha, \gamma+1 / 2} \psi_{i}\left(x_{\alpha, \gamma+1 / 2}\right)}{\rho_{i, \gamma+1 / 2} \Delta x \Delta z_{i, \gamma+1 / 2}},
\end{aligned}
$$

for diagnostic purposes such as analysis of divergence and vorticity fields (Frank et al., 2002). We illustrate the moving meshes in response to the generation of an orographic wave associated with an isolated hill in the vertical-slice model (Figure 1). If there is a disturbance that generates imbalance in the vertically stratified layers, the meshes move vertically to find new positions $z_{i, \gamma+1 / 2}^{n+1}$ that satisfy hydrostatic balance. The particles lie midway between adjacent horizontal meshes so that they also adjust vertically, conforming to the buoyant meshes. A similar moving-mesh approach was combined with an Eulerian finite-volume scheme and semi-Lagrangian advection in Lin (2004).

We did not use numerical diffusion for tests in this study, but spurious small-scale perturbations are filtered using a

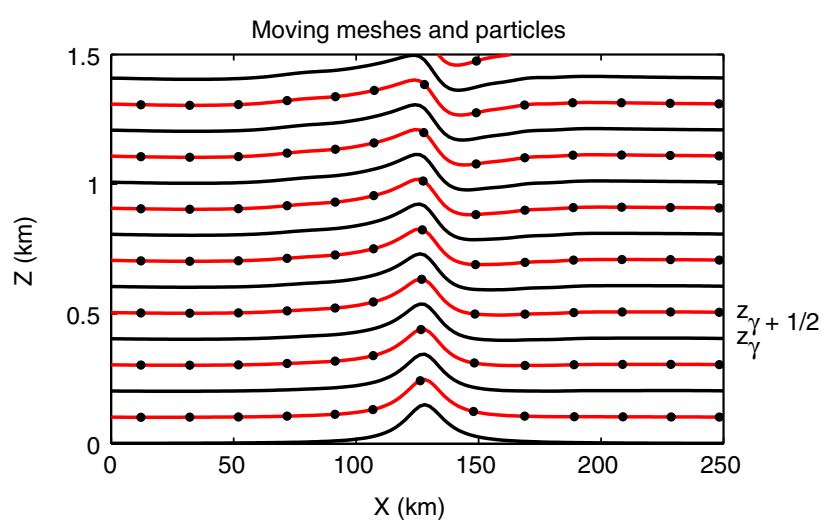

Figure 1. Schematic descriptions of particles conforming to a moving mesh due to an isolated hill. Vertical grids $\left(z_{\gamma}\right)$ are denoted by black lines and the mean of two vertically moving meshes, $z_{i, \gamma+1 / 2}=\left(z_{i, \gamma+1}+z_{i, \gamma}\right) / 2$ by shaded lines (red in the online article). We plot every 20 th particle (black dots) in each layer. This figure is available in colour online at wileyonlinelibrary.com/journal/qj
Helmholtz operator on the thermodynamic quantities over the grid (Frank et al., 2002). The Helmholtz operator is applied directly in the Lagrangian such that Hamiltonian structure is maintained. In the current context, we smooth $\bar{\rho}, \bar{\mu}, z$ and the Exner function $\pi$. Frank et al. (2005) showed that the combination of this regularization and Störmer-Verlet time-stepping modifies the dispersion relation in a manner equivalent to a two-time-level semiimplicit time discretization for the linearized shallow-water equations. Let $H_{i}^{i^{\prime}}$ denote a discrete approximation to the Helmholtz operator

$$
H=1-\alpha_{x}^{2} \frac{\partial^{2}}{\partial x^{2}}
$$

over the $x$ grid, subject only to $x$ and its periodic boundary conditions. Here $\alpha_{x} \geq 0$ is a given smoothing length. Then a smoothed $\tilde{\mu}_{i}$ is defined as the solution of

$$
\sum_{i^{\prime}} H_{i}^{i^{\prime}} \tilde{\mu}_{i^{\prime}}=\bar{\mu}_{i}
$$

Likewise we obtain $\widetilde{\rho}$ and $\widetilde{z}$ using the Helmholtz operator. Note that now $\widetilde{z}, \tilde{\mu} / \Delta z_{i, \gamma+1 / 2}$ and $\tilde{\rho} / \Delta z_{i, \gamma+1 / 2}$ replace $z, \mu$ and $\rho$ in (2.10). The associated force fields (2.18) and (2.19) are then given by

$$
\widetilde{F}_{x, \alpha, \gamma+1 / 2}^{\mathrm{G}}=-g m_{\alpha, \gamma+1 / 2} \sum_{i}\left[\widetilde{z}_{i, \gamma+1 / 2} \nabla_{x} \psi_{i}\left(x_{\alpha, \gamma+1 / 2}\right)\right]
$$

and

$$
\begin{aligned}
\widetilde{F}_{x, \alpha, \gamma+1 / 2}^{\mathrm{P}}=- & c_{\mathrm{p}} m_{\alpha, \gamma+1 / 2} \theta_{\alpha, \gamma+1 / 2} \\
& \times \sum_{i}\left[\tilde{\pi}_{i, \gamma+1 / 2} \nabla_{x} \psi_{i}\left(x_{\alpha, \gamma+1 / 2}\right)\right],
\end{aligned}
$$

where $\tilde{\pi}_{i}$ is defined as the solution of $\sum_{i^{\prime}} H_{i}^{i^{\prime}} \tilde{\pi}_{i^{\prime}}=$ $\left[\tilde{\mu} /\left(\mu_{0} \Delta z_{i, \gamma+1 / 2}\right)\right]^{\kappa /(1-\kappa)}$.

\section{Numerical experiments with orography}

We evaluate the performance of our scheme by testing the generation of gravity waves due to an idealized orography. Details of the experiments are listed briefly in Table I. The aim of these tests is to examine whether the hydrostatic HPM method is capable of capturing general features of gravity waves in the presence of orography. For this purpose we use the tests in the linear hydrostatic flow regime reported in DK83 and P95. As is pointed out in a number of references in the literature, an appropriate upper boundary layer is essential in numerical simulations of orographic flow to prevent the reflection of waves back into the domain. The portion of the domain where a dissipative boundary condition is put into operation is often called a 'sponge layer'. In our experiments we apply such a boundary condition only to the zonal wind. The sponge layer in DK83 is defined by

$$
U^{+}=U^{-}+\frac{\Delta t}{2} \tau(z)\left(U^{-}-U_{0}\right)
$$

where $U^{-}$is an updated zonal wind of a particle using (2.17), $U_{0}$ a constant zonal wind speed given at the initial time, 
Table I. Description of numerical experiments.

\begin{tabular}{lll}
\hline Test & Details & Description \\
\hline T1 & $U=20 \mathrm{~m} \mathrm{~s}^{-1}, a=10 \mathrm{~km}, N=0.0196 \mathrm{~s}^{-1}$ & Linear hydrostatic flow in DK83 \\
T2 & $U=32 \mathrm{~m} \mathrm{~s}^{-1}, a=16 \mathrm{~km}, N=0.0187 \mathrm{~s}^{-1}$ & Linear hydrostatic flow in P95 \\
T3 & $U=20 \mathrm{~m} \mathrm{~s}^{-1}, a=10 \mathrm{~km}, N=0.0132 \mathrm{~s}^{-1}$ & Linear dry $/$ moist flow in DK83 \\
T4 & $U=20 \mathrm{~m} \mathrm{~s}^{-1}, a=10 \mathrm{~km}, N=0.0132 \mathrm{~s}^{-1}$ & Nonlinear dry $/$ moist flow with $h_{0}=1 \mathrm{~km}$ \\
\hline
\end{tabular}

$\Delta t$ a time interval and $U^{+}$a new velocity. In DK83, $\tau(z)$ is defined by

$$
\tau(z)= \begin{cases}0 & \text { for } z \leq z_{\mathrm{B}}, \\ -\frac{\chi}{2}\left(1-\cos \frac{z-z_{\mathrm{B}}}{z_{\mathrm{T}}-z_{\mathrm{B}}} \pi\right) & \text { for } 0 \leq \frac{z-z_{\mathrm{B}}}{z_{\mathrm{T}}-z_{\mathrm{B}}} \leq 0.5, \\ -\frac{\chi}{2}\left[1+\left(\frac{z-z_{\mathrm{B}}}{z_{\mathrm{T}}-z_{\mathrm{B}}}-\frac{1}{2}\right) \pi\right] & \text { for } 0.5 \leq \frac{z-z_{\mathrm{B}}}{z_{\mathrm{T}}-z_{\mathrm{B}}} \leq 1,\end{cases}
$$

where $\chi$ is a constant, $z_{\mathrm{T}}$ is the top of the domain and $z_{\mathrm{B}}$ is the bottom of the sponge layer. We choose the constant $\chi=20 \mathrm{~h}^{-1}$, with which an optimal solution is produced in the experiments of this study. We have observed excessive dissipation or instability with values respectively much larger or smaller than this. Meanwhile, the type of sponge layer in P95 is given such that

$$
U^{+}=(1-\beta) U^{-}+\beta U_{0} .
$$

The coefficient $\beta=\beta_{\mathrm{v}}$ for the vertical sponge layer in P95 is defined by

$$
\beta_{\mathrm{v}}= \begin{cases}0 & \text { for } z<z_{\mathrm{B}} \\ \left(\frac{z-z_{\mathrm{B}}}{z_{\mathrm{T}}-z_{\mathrm{B}}}\right)^{2} & \text { for } z_{\mathrm{B}}<z<z_{\mathrm{T}}\end{cases}
$$

and similarly the coefficient $\beta=\beta_{\mathrm{h}}$ for lateral sponge zones is given by

$$
\beta_{\mathrm{h}}= \begin{cases}\cos ^{2}\left[\frac{\pi}{2}\left(\frac{x}{x_{\mathrm{D}}}\right)\right] & \text { for } 0<x<x_{\mathrm{D}} \\ \cos ^{2}\left[\frac{\pi}{2}\left(\frac{x_{\mathrm{L}}-x_{\mathrm{D}}}{x_{\mathrm{D}}}\right)\right] & \text { for } x_{\mathrm{L}}-x_{\mathrm{D}}<x<x_{\mathrm{L}} \\ 0 & \text { otherwise, }\end{cases}
$$

where $x_{\mathrm{L}}$ is the lateral size of the domain and $x_{\mathrm{D}}$ is the thickness of the sponge zone. We choose the P95 type of lateral sponge zone, since we obtain an optimal solution close to the analytic solution with this boundary condition. The Coriolis parameter is set to be zero for all tests. We use a bell-shaped profile for the orography, represented by

$$
h(x)=h_{0}\left(\frac{a^{2}}{x+a^{2}}\right) .
$$

Initially we begin with the peak height $h_{0}=0$ and increase $h_{0}$ gradually during the early stage of the integration until $h_{0}=1 \mathrm{~m}$. The isolated hill is placed at the centre of the domain. The number of particles is two per cell and the total number of particles per layer is kept uniform.

\subsection{T1: Linear hydrostatic flow}

Figure 2 shows the grid-based zonal wind perturbation $\left(u^{\prime}\right.$ $\left.=u-U_{0}\right)$ and the vertical wind perturbation $\left(w^{\prime}=w\right)$. The size of the spatial domain is $L_{x}=180 \mathrm{~km}$ and $L_{z}=16 \mathrm{~km}$ and the atmosphere is isothermal with temperature $T_{0}=250 \mathrm{~K}$ as in DK83. The bottom of the sponge layer $z_{\mathrm{B}}$ is $8 \mathrm{~km}$. For the lateral sponge zone, we choose the thickness $x_{\mathrm{L}}=2 \mathrm{~km}$. The smoothing length $\alpha_{x}=1 \mathrm{~km}$. The spatial resolution in DK83 was $2 \mathrm{~km}$, but we choose it to be $1 \mathrm{~km}$ since we obtain a more accurate solution with the doubled spatial resolution. The vertical velocity perturbation is better captured in our hydrostatic model with the increase in spatial resolution. We can integrate the equation with a time-step size of $18 \mathrm{~s}$. Figure 2 also shows time tendency of the energy and the profile of the vertical flux of horizontal momentum $M$, which is defined by

$$
M=\int_{-\infty}^{\infty} \rho u^{\prime} w^{\prime} \mathrm{d} x
$$

in terms of Reynolds stress, and this is normalized by the pressure drag on the surface, approximated by

$$
D=\frac{-\pi}{4} \rho_{0} N U_{0} h_{0}^{2}
$$

for a linear mountain wave (Durran and Klemp, 1983). As shown in (3.8), the buoyancy frequency $N$ controls the magnitude of the surface drag given the mountain height and mean wind speed. The simulation is stable, as implied by the energy tendency, and the wave structure and magnitude of the perturbation are in good agreement with the reference solution presented in DK83. For example, the local minimum of the zonal wind perturbation around $z=5 \mathrm{~km}$ is correctly captured, as seen by the contour line of $-24 \times 10^{-3} \mathrm{~m} \mathrm{~s}^{-1}$ (see figure 1a in DK83 for the analytic solution). The normalized momentum flux during the quasi-steady state is about 0.998 (Figure 2) and this is quite close to the theoretical value of 1 . We observe that the coefficient $\chi$ for the vertical sponge layer influences the magnitude of the perturbation and the behaviour of the vertical momentum flux (not shown). The larger $\chi$ becomes, the weaker the momentum flux.

\subsection{T2: Linear hydrostatic flow in $P 95$}

The height of the hill $h_{0}$ is the same as DK83, but the hill is broader and the uniform initial wind speed is stronger (32 $\mathrm{m} \mathrm{s}^{-1}$ instead of $20 \mathrm{~m} \mathrm{~s}^{-1}$ ). Also the domain size is larger, with $L_{x}=512 \mathrm{~km}$ and $L_{z}=20 \mathrm{~km}$. We use the same spatial resolution $\Delta x=3.2 \mathrm{~km}$ and $\Delta z=0.25 \mathrm{~km}$ as in P95. In our simulations $\Delta t$ can be up to $36 \mathrm{~s}$, with the smoothing scale $\alpha_{x}=3.2 \mathrm{~km}$ for the result shown in this article. In 

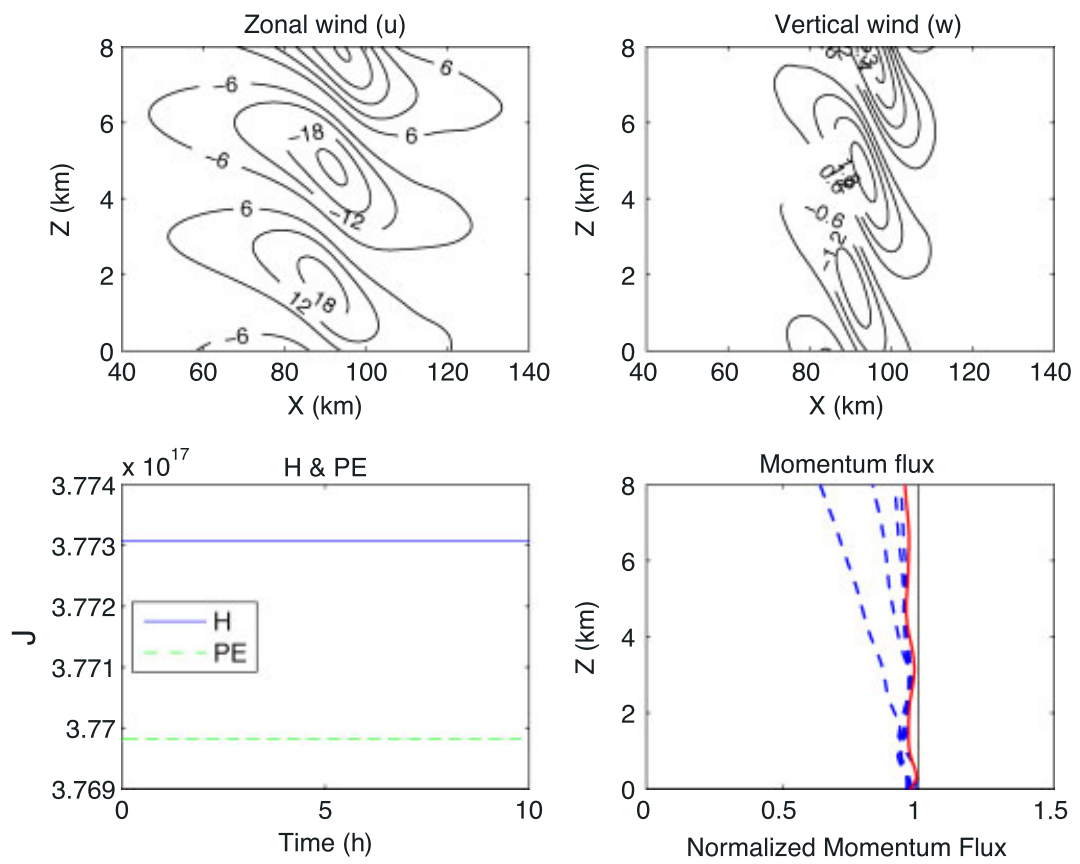

Figure 2. The zonal wind $\left(u^{\prime}\right)$, vertical velocity $\left(w^{\prime}\right)$, time tendency of the total energy $(H)$ and potential energy $(\mathrm{PE})$ and the vertical profile of the normalized momentum flux. We display here $1000 u^{\prime}$ and $1000 w^{\prime}$. The contour interval is $6 \times 10^{-3} \mathrm{~m} \mathrm{~s}^{-1}$ for the zonal wind and $0.6 \times 10^{-3} \mathrm{~m} \mathrm{~s}^{-1}$ for the vertical wind. They are the solution at $t=10 \mathrm{~h}$, when it is quasi-steady. The momentum flux at $10 \mathrm{~h}$ is shaded (red in the online article) and the flux at earlier integration times displayed in dashed lines (blue in the online article). See text for more details. This figure is available in colour online at wileyonlinelibrary.com/journal/qj

P95, $z_{\mathrm{B}}$ is not explicitly given, but they suggest that $z_{\mathrm{B}}=L_{z}$ $-\lambda_{z}$, where $\lambda_{z}$ is the vertical wavelength of the dominant wave. The wavelength is $\lambda_{z}=2 \pi U_{0} / N$ so that the bottom of the sponge layer $z_{\mathrm{B}}$ is estimated to be $9.29 \mathrm{~km}$. For the lateral sponge zone, we choose the thickness $x_{\mathrm{L}}=2 \mathrm{~km}$ as in T1. Figure 3(a) shows the result from the experiment with the P95 type of vertical sponge layer and Figure 3(b) shows the result with the DK83 type of sponge layer. In both simulations we use the P95 type of lateral sponge zone. The wave structure is similar in each case and also similar to the reference solution in P95, but the magnitude of the perturbation in Figure 3(a) is slightly weaker. The momentum flux is well resolved in both experiments and close to the analytical estimation.

\section{Representation of moist processes}

We consider here highly simplified condensation and evaporation processes explicitly resolved over particles. We summarize the notation for the variables associated with moist processes:

$m$

$m_{\mathrm{v}} \quad$ mass of water-vapour component

$m_{\mathrm{c}}$ mass of liquid water component

$r_{\mathrm{v}}$ mixing ratio of water vapour, $r_{\mathrm{v}}=m_{\mathrm{v}} / m_{\mathrm{d}}$

$r_{\mathrm{c}}$ cloud water, $r_{\mathrm{c}}=m_{\mathrm{c}} / m_{\mathrm{d}}$

$r_{\mathrm{s}}$ mixing ratio of liquid water

$r_{\mathrm{t}}$ total moisture content of an air parcel, $r_{\mathrm{t}}=r_{\mathrm{v}}+r_{\mathrm{c}}$

$L$ latent heat of vaporization at $0^{\circ} \mathrm{C}=2.5 \times 10^{6} \mathrm{~J} \mathrm{~kg}^{-1}$

$R_{\mathrm{V}} \quad$ specific gas constant of water vapour $=461.5 \mathrm{~J} \mathrm{~kg}^{-1} \mathrm{~K}^{-1}$
To account for liquid water loading and water vapour in the reversible moist process, the ideal gas equation in the moist environment can be expressed by

$$
p=\rho R_{\mathrm{d}} T \frac{1+r_{\mathrm{v}} / \epsilon}{1+r_{\mathrm{t}}},
$$

where $\epsilon=R_{\mathrm{d}} / R_{\mathrm{v}} \approx 0.622$, the ratio of the gas constant of dry air to water vapour. Accordingly, hydrostatic balance is defined by

$$
\frac{\mathrm{d} \pi}{\mathrm{d} z}=-\frac{g}{c_{\mathrm{p}} \theta} \frac{1+r_{\mathrm{t}}}{1+r_{\mathrm{v}} / \epsilon} .
$$

The relation between $\pi$ and $\mu=\rho \theta$ becomes

$$
\pi=\left[\frac{\mu}{\mu_{0}}\left(\frac{1+\frac{r_{\mathrm{v}}}{\epsilon}}{1+r_{\mathrm{t}}}\right)\right]^{\kappa /(1-\kappa)} .
$$

Given an initial condition, the density over the position of a particle $\mathbf{x}_{\alpha, \gamma+1 / 2}$ can be approximated by

$$
\rho_{\alpha, \gamma+1 / 2}=\frac{p_{\alpha, \gamma+1 / 2}}{R T_{\alpha, \gamma+1 / 2}} \frac{1+r_{v_{\alpha, \gamma+1 / 2}} / \epsilon}{1+r_{t_{\alpha, \gamma+1 / 2}}} .
$$

We assign the (total) mass of the 'moist particle' as $m_{\alpha, \gamma+1 / 2}$ $\approx \rho_{\alpha, \gamma+1 / 2}(t=0) \Delta a \Delta c$. This is done at the initial time and the mass of the particle remains unchanged in time. Using the hydrostatic equation (4.2), we calculate the vertical mesh position $z_{i, \gamma+1 / 2}$ and the pressure gradient force for the particles is given by

$$
\begin{gathered}
F_{x, \alpha, \gamma+1 / 2}^{\mathrm{P}}=-c_{\mathrm{p}} m_{\alpha, \gamma+1 / 2} \theta_{\alpha, \gamma+1 / 2} \\
\times \sum_{i}\left[\left(\frac{\mu_{i, \gamma+1 / 2}}{\mu_{0}} \frac{1+r_{v_{i, \gamma+1 / 2}} / \epsilon}{1+r_{t_{i, \gamma+1 / 2}}}\right)^{\kappa /(1-\kappa)} \nabla_{x} \psi_{i}\left(x_{\alpha, \gamma+1 / 2}\right)\right] .
\end{gathered}
$$



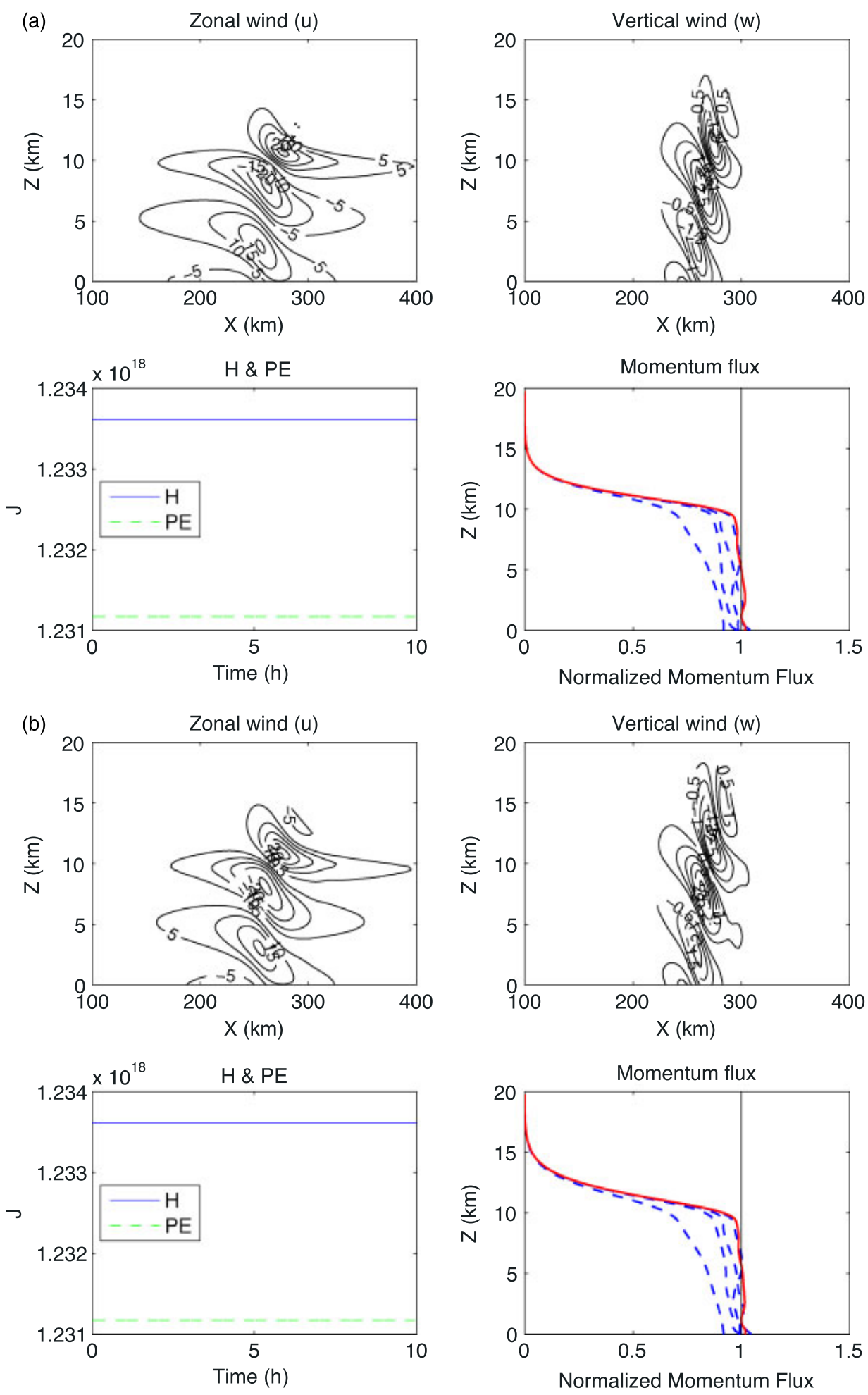

Figure 3. The same as Figure 2, except that the contour interval is $5 \times 10^{-3} \mathrm{~m} \mathrm{~s}^{-1}$ for the zonal wind and $0.5 \times 10^{-3} \mathrm{~m} \mathrm{~s}^{-1}$ for the vertical wind. (a) T2 with the vertical sponge layer of P95. (b) T2 with the vertical sponge layer of DK83. The P95 type of lateral sponge zone is used in both simulations. This figure is available in colour online at wileyonlinelibrary.com/journal/qj

Even in the absence of phase changes, $r_{s_{\alpha, \gamma+1 / 2}}$ on the particles at each time $t$ needs to be calculated if the pressure and temperature approximated at the position have been changed. We use Bolton's formula (1980) for the saturated vapour pressure:

$$
e_{s_{\alpha, \gamma+1 / 2}}=6.11 \exp \left[\frac{17.67\left(T_{\alpha, \gamma+1 / 2}-273\right)}{243.5+T_{\alpha, \gamma+1 / 2}-273}\right] \text {, }
$$

where $e_{\mathrm{s}}$ is in $\mathrm{hPa}$. Then we obtain the saturation mixing ratio $r_{\mathrm{s}}$ :

$$
r_{s_{\alpha, \gamma+1 / 2}} \approx \epsilon \frac{e_{s_{\alpha, \gamma+1 / 2}}}{\left(p_{\alpha, \gamma+1 / 2}-e_{s_{\alpha, \gamma+1 / 2}}\right)} .
$$

The thermodynamic equations for the phase conversion processes are similar to those for the moisture budget in Klemp and Wilhelmson (1978), but we do not 

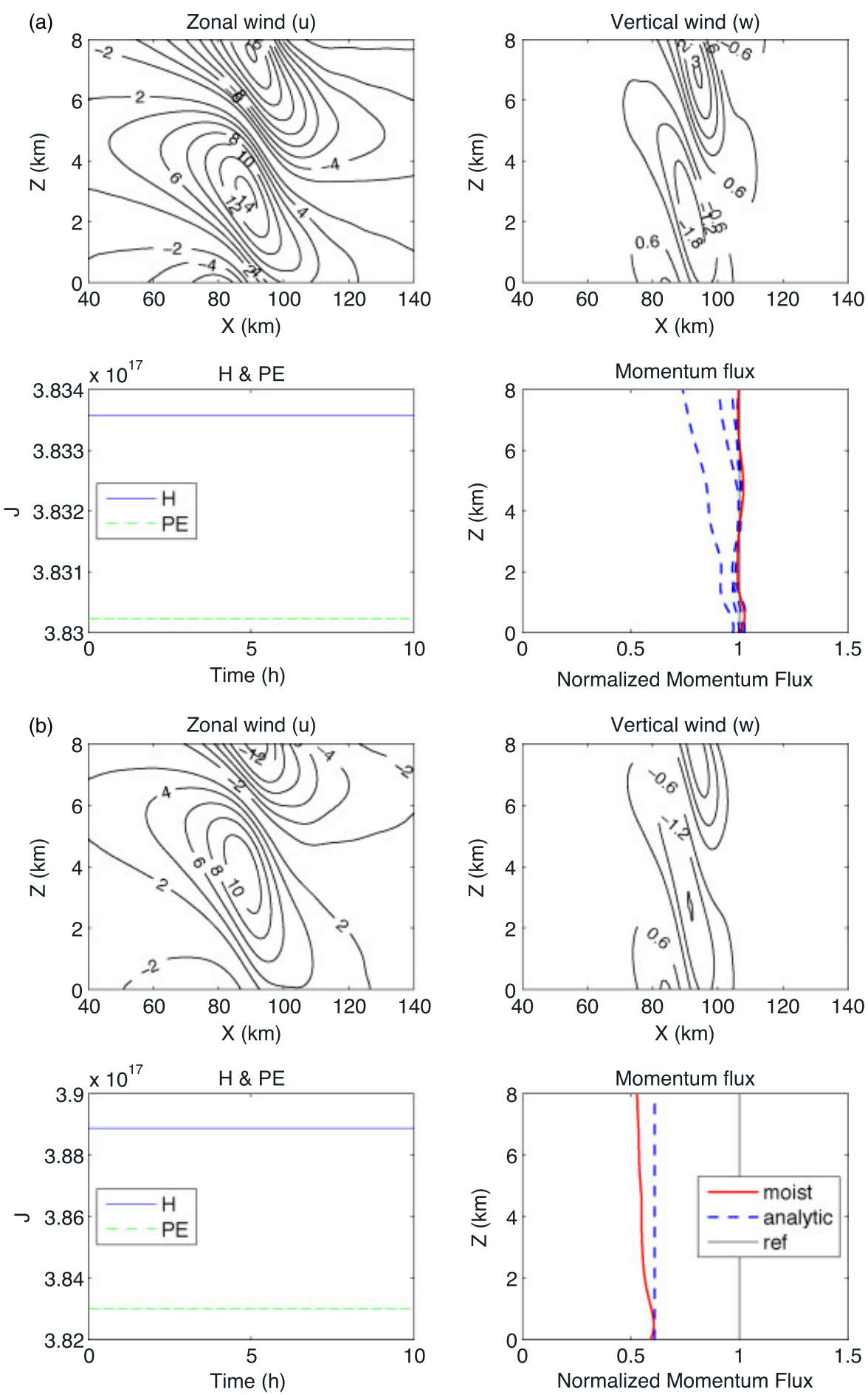

Figure 4. The same as Figure 2, except for some parameters given for the initial set-up. (a) Dry simulation with RH $=0 \%$ and surface temperature $T_{0}=273 \mathrm{~K}$. (b) The same as (a), but $\mathrm{RH}=100 \%$. (c) $\mathrm{RH}=100 \%$ and $T_{0}=280 \mathrm{~K}$. See text for details. This figure is available in colour online at wileyonlinelibrary.com/journal/qj

include any numerical diffusion terms or subgrid turbulent parametrization. The prognostic equations for the two moist components -water vapour and condensed liquid water -and the potential temperature are given by

$$
\begin{aligned}
\frac{\mathrm{d} r_{\mathrm{v}}}{\mathrm{d} t} & =\delta\left(r_{\mathrm{v}}, r_{\mathrm{c}}\right) \frac{\mathrm{d} r_{\mathrm{s}}}{\mathrm{d} t}, \\
\frac{\mathrm{d} r_{\mathrm{c}}}{\mathrm{d} t} & =-\delta\left(r_{\mathrm{v}}, r_{\mathrm{c}}\right) \frac{\mathrm{d} r_{\mathrm{s}}}{\mathrm{d} t}, \\
\frac{\mathrm{d} \ln \theta}{\mathrm{d} t} & =-\frac{L}{c_{\mathrm{p}} T} \frac{\mathrm{d} r_{\mathrm{v}}}{\mathrm{d} t} .
\end{aligned}
$$

The Heaviside function $\delta\left(r_{\mathrm{v}}, r_{\mathrm{c}}\right)$ has been introduced to represent phase conversions that occur under the conditions

$$
\delta\left(r_{\mathrm{v}}, r_{\mathrm{c}}\right)= \begin{cases}1 & \text { for } r_{\mathrm{v}} \geq r_{\mathrm{s}} \text { or } r_{\mathrm{c}}>0 \\ 0 & \text { for } r_{\mathrm{v}}<r_{\mathrm{s}} \text { and } r_{\mathrm{c}}=0 .\end{cases}
$$

We assume that the phase conversions take place over each particle and the moist components compose the total mass of the particle. The latent heat of vaporization $(L)$ does indeed vary with the temperature, but we take it as constant for the numerical experiments, where the effect is negligible 

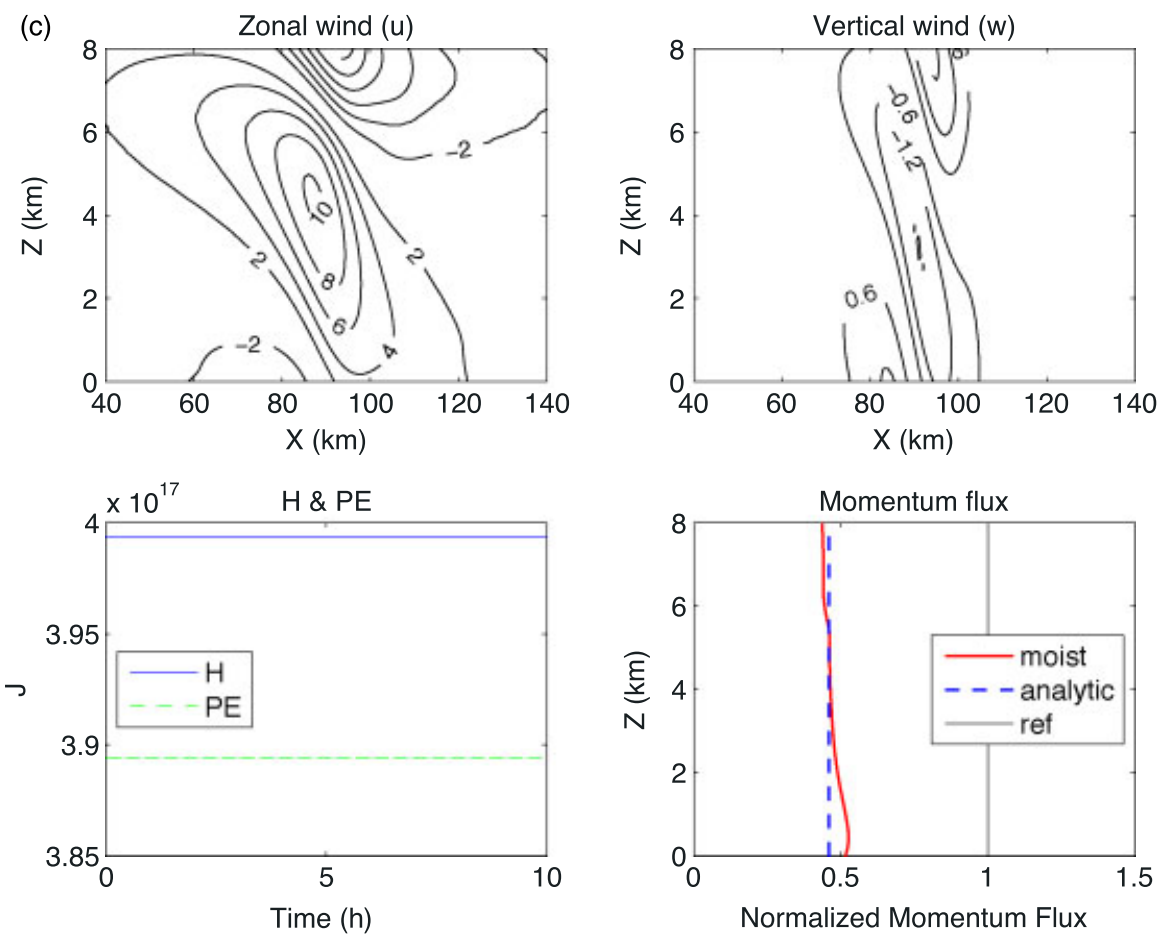

Figure 4. (Continued) This figure is available in colour online at wileyonlinelibrary.com/journal/qj

for the purpose of this study. The total water content $r_{\mathrm{t}}$ is conserved in the absence of rain and turbulent mixing.

Once the parcel is saturated, $r_{\mathrm{v}}-r_{\mathrm{s}}>0$, the excess is converted into liquid water and added to $r_{\mathrm{c}}$. The new $r_{\mathrm{v}}$ becomes equal to $r_{\mathrm{s}}$. The change of saturation mixing ratio $\Delta r_{\mathrm{s}}$ and the associated change of the temperature $\Delta T$ through phase conversion is approximated by

$$
\begin{aligned}
& \Delta r_{\mathrm{s}} \approx \frac{r_{\mathrm{s}}-r_{\mathrm{v}}}{1+L^{2} r_{\mathrm{s}} /\left(c_{\mathrm{p}} R_{\mathrm{v}} T^{2}\right)}, \\
& \Delta T \approx-\frac{L \Delta r_{\mathrm{s}}}{c_{\mathrm{p}}} .
\end{aligned}
$$

The derivation of the equation for $\Delta r_{\mathrm{s}}$ is described in Appendix A. To ensure the values of $r_{\mathrm{c}}$ and $r_{\mathrm{v}}$ remain nonnegative and $r_{\mathrm{t}}$ is invariant, we limit $\Delta r_{\mathrm{s}}=\max \left(\Delta r_{\mathrm{s}}, r_{\mathrm{v}}\right)$ for condensation and $\Delta r_{\mathrm{s}}=\min \left(\Delta r_{\mathrm{s}},-r_{\mathrm{c}}\right)$ for evaporation. Subsequently, we can update the mixing ratio of water vapour, liquid water and potential temperature using (4.8). Then, the mesh height $z_{i, \gamma+1 / 2}$ is calculated to satisfy a new hydrostatic balance after the occurrence of phase changes. To be consistent with (4.2) and (4.8), the energy $(\mathcal{E}=\mathcal{V}+\mathcal{T}+\mathcal{Q})$ of the system has contributions

$$
\begin{aligned}
\mathcal{V}= & \sum_{i, \gamma}\left[c_{\mathrm{v}} \mu_{0}\left[\frac{\mu_{i, \gamma+1 / 2}}{\mu_{0}}\left(\frac{1+r_{v_{i, \gamma+1 / 2}} / \epsilon}{1+r_{t_{i, \gamma+1 / 2}}}\right)\right]^{1 /(1-\kappa)}\right. \\
& \left.+g \rho_{i, \gamma+1 / 2} z_{i, \gamma+1 / 2}\right] \Delta x \Delta z_{i, \gamma+1 / 2} \\
\mathcal{T} & =\frac{1}{2} \sum_{\alpha, \gamma} m_{\alpha, \gamma+1 / 2}\left|\dot{x}_{\alpha, \gamma+1 / 2}\right|^{2} \\
\mathcal{Q} & =L \sum_{\alpha, \gamma} r_{v_{\alpha, \gamma+1 / 2}} m_{\alpha, \gamma+1 / 2}
\end{aligned}
$$

4.1. T3: Linear dry/moist hydrostatic flow compared with DK83

As described in the literature (e.g. DK83), the strength of the orographic wave can diminish when moisture is present. The temperature profile is calculated given stability $N=0.0132$ $\mathrm{s}^{-1}$ with surface temperature $T_{0}=273 \mathrm{~K}$. The approximate Scorer parameter $l=\left[N^{2} / U_{0}^{2}-\left(\partial^{2} U_{0} / \partial^{2} z\right) / U_{0}\right]^{1 / 2}$ can be constant with height, as discussed in DK83. For test T3 we use the same numerical set-up as for test T1, except $\Delta t=9 \mathrm{~s}$. To obtain a stable moist solution in the test with a higher surface temperature than $273 \mathrm{~K}$, a smaller time-step size than $\Delta t=18 \mathrm{~s}$ is required. Figure 4(a) shows the result from the dry simulation and Figure 4(b) shows the result from the moist simulation with relative humidity $\mathrm{RH}=100 \%$ with $r_{\mathrm{c}}=0$ initially. In comparison with the dry simulation with $\mathrm{RH}=0 \%$ (Figure $4(\mathrm{a})$ ), the wavelength is longer and the perturbation is weaker. The theoretical vertical wavelength $\lambda_{z}=2 \pi U / N$ might increase as the stability is reduced due to the presence of moist processes. We approximate the first vertical half-wavelength of numerical solutions by examining the height at which the contour of the isentropic surface becomes a mirror image of the isolated hill (DK83). The first vertical half-wavelength is about $4.5 \mathrm{~km}$ in the test without moisture $(\mathrm{RH}=0 \%)$, while it is about $7.5 \mathrm{~km}$ in the moist simulation. As in DK83, we compare the momentum flux in this test with an analytical estimation using the approximate moist stability $N_{\mathrm{m}}$ suggested in Durran and Klemp (1982). For comparison, we denote the reference magnitude of momentum flux of the dry case by $D$, defined in (3.8). The approximate moist stability is $N_{\mathrm{m}}=0.008 \mathrm{~s}^{-1}$ at the surface given $T_{0}=273 \mathrm{~K}$ and $\mathrm{RH}=100 \%$. The momentum flux can be reduced to $0.61 D$ theoretically, if $N_{\text {m }}$ replaces $N$ in (3.8). The vertical mean $(0 \sim 8 \mathrm{~km})$ of the momentum flux in Figure 4(a) is about $0.56 \mathrm{D}$, which is lower than the expected value of $0.61 D$. In DK83, note that the momentum flux is about $0.4 \mathrm{D}$ instead of the theoretical 

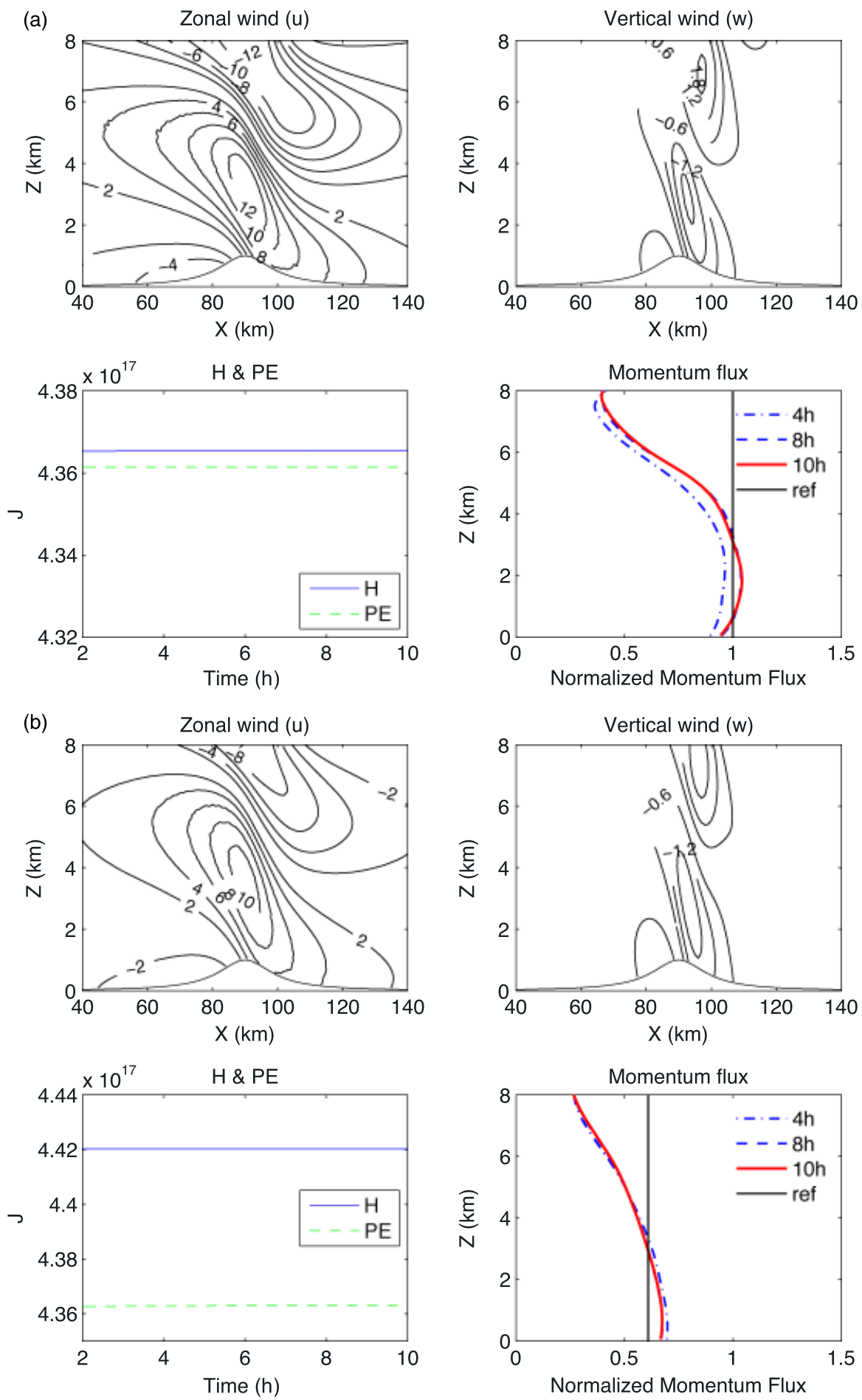

Figure 5. The same as Figure 4(a) and (b), except that $h_{0}=1 \mathrm{~km}, L_{z}=32 \mathrm{~km}$ and the contour interval is $6 \mathrm{~m} \mathrm{~s}^{-1}$ for zonal wind and $0.6 \mathrm{~m} \mathrm{~s}{ }^{-1}$ for vertical wind perturbation. This figure is available in colour online at wileyonlinelibrary.com/journal/qj

estimation $0.47 \mathrm{D}$ in the partially cloudy case, while the difference is smaller in the 'everywhere cloudy' case in DK83. In our simulation it is cloudy in the layer below about $5 \mathrm{~km}$. To examine the effect of the cloudiness we retain the stability $N=0.0132 \mathrm{~s}^{-1}$, but increase the surface temperature to $280 \mathrm{~K}$ so that more latent heat can be released. This initial set-up yields the approximate moist stability $N_{\mathrm{m}}=0.006 \mathrm{~s}^{-1}$ and the depth of the cloudy layer extends to $6.5 \mathrm{~km}$. The analytic estimation of surface drag is reduced to $0.46 \mathrm{D}$ with the decreased stability. The vertical mean of the momentum flux is about $0.47 D$ in our simulation (Figure $4(c)$ ), which shows that the vertical profile of momentum flux becomes closer to the theoretical analysis as cloudiness increases. Those results from T3 experiments indicate that the effect of moist processes on the characteristics of a mountain wave is reasonably represented by the hydrostatic HPM.

\subsection{T4: Nonlinear dry/moist hydrostatic flow}

We use the same initial set-up as for the first two tests in T3, except that $h_{0}=1 \mathrm{~km}, \Delta t=18 \mathrm{~s}$ and the vertical domain size $L_{z}=32 \mathrm{~km}$ for the nonlinear test. We increase the domain size to implement a very thick sponge layer 
for highly nonlinear mountain waves (Durran and Klemp, 1983), but maintain the same vertical resolution as in T3. The height of the mountain is increased from zero to $1 \mathrm{~km}$ gradually for an hour to avoid the generation of instabilities. During this period, we also add a frictional damping,

$$
\begin{aligned}
F_{\alpha, \gamma+1 / 2}^{\mathrm{F}}=-\beta m_{\alpha, \gamma+1 / 2}\left(\dot{x}_{\alpha+1, \gamma+1 / 2}\right. & \\
& \left.-2 \dot{x}_{\alpha, \gamma+1 / 2}+\dot{x}_{\alpha-1, \gamma+1 / 2}\right),
\end{aligned}
$$

to the right-hand side of the momentum equation (2.17). A damping coefficient $\beta=2 / \Delta t$ is used in this test. Since the damping is applied only while the height is increased, the variational relation would not be affected after the height of mountain reaches $1 \mathrm{~km}$. The momentum flux is normalized by $D_{n}=-(\pi / 4) \rho_{0} N U_{0} h_{0}^{2}\left[1+(7 / 16)\left(h_{0} l\right)^{2}\right]$ (Miles and Huppert, 1969) instead of (3.8). Figure 5(a) shows that the dry solution is stable and represents a similar wave structure to that of the linear counterpart (Figure 4(a)). However, the momentum flux diverges with height, especially above $4 \mathrm{~km}$ where streamline reversal occurs. Non-hydrostatic effects may play an important role in this highly nonlinear case (Durran and Klemp, 1983) and the vertical transport of momentum might not be adequately represented by a hydrostatic model. Durran (1995) suggests that the waveinduced momentum fluxes need to be parametrized for a complete description of subgrid-scale forcing related to wave propagation. Such aspects would certainly require further investigation. Figure 5(b) shows that the nonlinear moist wave is weakened by latent heat releases, as in the linear test T3. The results from the nonlinear tests show that our model performs stably in the more challenging nonlinear case, and produces wave structures that are qualitatively similar to those of the linear cases.

\section{Discussion and outlook}

We have extended the HPM method to a hydrostatic vertical-slice model and evaluated its performance using some idealized tests of both dry and moist atmospheres. It is shown that the model captures the fundamental features of an orographic gravity wave and the results are quantitatively in good agreement with reference solutions. In particular, moist processes are newly implemented in an HPM model and the moist effect on the gravity wave is reasonably well reproduced. We show the potential of the hydrostatic HPM for a dynamical core suitable for climate simulations, where conservation of mass of air, water and long-lived tracers is essential and no artefactual generation of energy is desirable.

As the method stands, particles adjust vertically conforming to the moving mesh. However, we may need to consider a redistribution of particles when diabatic processes lead to convective instability. A simple solution might be vertically exchanging particles between layers dependent on heating/cooling, to resemble convection. In future we will test such vertical exchanges of particles, aiming to develop a convective parametrization method for the hydrostatic HPM to describe cumulus convection and the response of largerscale circulations. One can anticipate in such situations that the horizontal velocity distribution of particles may tend to be non-uniform. To prevent this, we may need to include a diffusion mechanism. This implies that we leave the strictly Lagrangian variational approach, but it would add computational flexibility. We will investigate the technical aspect of particle remapping further in future publications.

\section{Acknowledgement}

We thank two anonymous reviewers for their suggestions and comments, which improved the manuscript.

\section{A. Appendix}

We use the simplified description of a moist process suggested in Haltiner and Williams (1980). The condensation/evaporation of water vapour should satisfy the following relation:

$$
r_{\mathrm{v}}+\Delta r_{\mathrm{v}}=r_{\mathrm{s}}(T+\Delta T, p)
$$

during the time interval $\Delta t$, assuming pressure is unchanged. This assumption is relevant for the hydrostatic regime. The term on the right-hand side can be approximated using the Taylor expansion

$$
r_{\mathrm{s}}(T+\Delta T, p) \approx r_{\mathrm{s}}(T)+\left(\frac{\partial r_{\mathrm{s}}}{\partial T}\right)_{p} \Delta T .
$$

Since

$$
r_{\mathrm{s}}=\epsilon\left(\frac{e_{\mathrm{s}}}{p}\right)
$$

and

$$
\frac{\mathrm{d} e_{\mathrm{s}}}{\mathrm{d} T}=\frac{L e_{\mathrm{s}}}{R_{\mathrm{v}} T^{2}}
$$

we rewrite the Clausius-Clapeyron equation in terms of $r_{\mathrm{s}}$ as

$$
\frac{\mathrm{d} r_{\mathrm{s}}}{\mathrm{d} T}=\frac{L r_{\mathrm{s}}}{R_{\mathrm{v}} T^{2}}
$$

Since $\Delta T=-L \Delta r_{\mathrm{v}} / c_{\mathrm{p}}$ by the thermodynamic law, we combine (A.1) and (A.2) and use (A.5) to obtain

$$
r_{\mathrm{v}}+\Delta r_{\mathrm{v}} \approx r_{\mathrm{s}}+\left(\frac{L r_{\mathrm{s}}}{R_{\mathrm{v}} T^{2}}\right)\left(\frac{-L \Delta r_{\mathrm{v}}}{c_{\mathrm{p}}}\right) .
$$

Thus,

$$
\Delta r_{\mathrm{v}} \approx r_{\mathrm{s}}-r_{\mathrm{v}}+\left(\frac{L r_{\mathrm{s}}}{R_{\mathrm{v}} T^{2}}\right)\left(\frac{-L \Delta r_{\mathrm{v}}}{c_{\mathrm{p}}}\right) .
$$

Solving this for $\Delta r_{\mathrm{v}}$ yields

$$
\Delta r_{\mathrm{v}} \approx \frac{r_{\mathrm{s}}-r_{\mathrm{v}}}{1+\left(\frac{L^{2} r_{\mathrm{s}}}{c_{\mathrm{p}} R_{\mathrm{v}} T^{2}}\right)} .
$$

\section{References}

Bolton D. 1980. The computation of equivalent potential temperature. Mon. Weather Rev. 108: 1046-1053.

Cotter C, Frank J, Reich S. 2004. Hamiltonian particle-mesh method for two-layer shallow-water equations subject to the ligid-lid approximation. SIAM J. Appl. Dyn. Sys. 3: 69-83.

Durran DR. 1995. Do breaking mountain waves decelerate the local mean flow? J. Atmos. Sci. 42: 4010-4032.

Durran DR, Klemp JB. 1982. On the effects of moisture on the Brunt-Väisälä frequency. J. Atmos. Sci. 39: 2152-2158. 
Durran DR, Klemp JB. 1983. A compressible model for the simulation of moist mountain waves. Mon. Weather Rev. 111: 2341-2361.

Frank J, Reich S. 2004. The Hamiltonian particle-mesh method for the spherical shallow water equations. Atmos. Sci. Lett. 5: 89-95.

Frank J, Gottwald G, Reich S. 2002. The Hamiltonian particle-mesh method. In Meshfree Methods for Partial Differential Equations, Lecture Notes in Computational Science and Engineering, Vol. 26, Griebel M, Schweitzer M (eds). Springer-Verlag: Berlin; pp 131-142.

Frank J, Reich S, Staniforth A, White A, Wood N. 2005. Analysis of a regularized, time-staggered discretization method and its link to the semi-implicit method. Atmos. Sci. Lett. 6: 97-104.

Haltiner GJ, Williams RT. 1980. Numerical Prediction and Dynamic Meteorology. John Wiley: New York, NY.
Klemp JB, Wilhelmson RB. 1978. The simulation of three-dimensional convective storm dynamics. J. Atmos. Sci. 35: 1070-1096.

Lin SJ. 2004. A 'vertically Lagrangian' finite-volume dynamical core for global models. Mon. Weather Rev. 132: 2293-2307.

Miles JW, Huppert HE. 1969. Lee waves in a stratified flow. Part IV: Perturbation approximation. J. Fluid. Mech. 35: 497-525.

Pinty J-P, Benoit R, Richard E, Laprise R. 1995. Simple tests of a semiimplicit semi-Lagrangian model on 2D mountain wave problems. Mon. Weather Rev. 123: 3042-3058.

Shin S, Reich S. 2009. Hamiltonian particle-mesh simulation for a non-hydrostatic vertical slice model. Atmos. Sci. Lett. 10: 233-240.

Thuburn J. 2008. Some conservation issues for the dynamical cores of NWP and climate models. J. Comput. Phys. 227: 3715-3730. 